\title{
The push-through total femoral prosthesis offers a functional alternative to total femoral replacement: a case series
}

\author{
Jelle Gorter $^{1}$ (D) - Joris J. W. Ploegmakers ${ }^{1}$ - Bas L. E. F. ten Have ${ }^{2}$. \\ Hendrik W. B. Schreuder ${ }^{3}$. Paul C. Jutte ${ }^{1}$
}

Received: 3 September 2016 / Accepted: 12 March 2017 / Published online: 30 March 2017

(C) The Author(s) 2017. This article is published with open access at Springerlink.com

\begin{abstract}
Purpose Oncologic resections or complications of segmental femoral prostheses can result in severe bone loss of the femur for which a total femoral prosthesis (TFP) is required. This study assesses whether the loss of stability and function caused by the loss of muscle attachments can be improved by using a push-through total femoral endoprosthesis (PTTF), because it saves parts of the femur and its muscle attachments. Methods In this retrospective case series, ten patients aged 25-77 (mean 54) who received a PTTF between 2005 and 2014 were included for baseline, complications and survival analysis with a mean follow-up of 5.3 (1.1-9.6) years. Functional outcome was assessed in six patients using the Musculoskeletal Tumor Society (MSTS) score, WHO performance scale, Toronto Extremity Salvage Score (TESS), SF36, EQ-5D, NRS pain score, fatigue score and satisfaction score. Results The mean MSTS score was 64\% (23-93\%). Five patients had a WHO performance scale of 1 , one patient of 3 . Mean TESS was 69\% (13-90\%). SF36 was most notably limited by physical functioning (mean 48), vitality (68) and general health (67). NRS score was 1.9, 1.8 and 8.3 for pain, fatigue and satisfaction, respectively. There were four failures: two infections (one resulting in amputation and one in a minor
\end{abstract}

Jelle Gorter

jellegorter@gmail.com

1 Department of Orthopaedic Surgery, University of Groningen, University Medical Center Groningen, P.O. Box 30001, 9700 RB Groningen, The Netherlands

2 Department of Orthopaedic Surgery, Martini Hospital, P.O. Box 30033, 9700 RM Groningen, The Netherlands

3 Department of Orthopaedic Surgery, Radboud University, Radboud University Medical Center, P.O. Box 9101, 6500 HB Nijmegen, The Netherlands revision) and two mechanical failures (which required one revision to a TFP and one minor revision). Patient survival was $100 \%$, limb survival $90 \%$, and prosthesis survival $80 \%$. Conclusion The push-through total femoral endoprosthesis allows preservation of muscle attachments and offers a good alternative to total femoral prostheses.

Keywords Push-through $\cdot$ Total femoral prosthesis $\cdot$ Limb salvaging $\cdot$ Functional outcome $\cdot$ Arthroplasty

\section{Introduction}

Overall outcome and survival rates of lower limb malignancies have improved rapidly since the 1970s due to improved (neo) adjuvant chemotherapy and limb-salvaging procedures [1-3]. However, the severe femoral bone loss and extensive soft tissue damage that is associated with limb-salvaging surgery and complications of arthroplasty required the development of megaprostheses [3]. The current megaprostheses offer satisfying results, however, complications such as infection or dislocation are common [4-8]. Proximal or distal femoral prostheses outperform total femoral prostheses (TFPs), which is often attributed to the conservation of the muscle insertions at the remaining femoral bone $[4,7,9]$. The integrity of the hip abductors and knee extensors affects the functional result of a TFP; hip dislocations occur more often after the abductor muscles are excised [10]. Attempts to fixate these muscles on the prosthesis have mostly been unsuccessful $[9,11]$.

The "Durchsteck" - or push-through - total femoral endoprosthesis (PTTF) (Figs. 1, 2, 3, and 4) distinguishes itself from the 'regular' TFP by connecting the knee component to the hip component with an intramedullary metal stem. This allows preservation of any remaining femoral cortex and its muscle attachments (Fig. 2), which should increase 

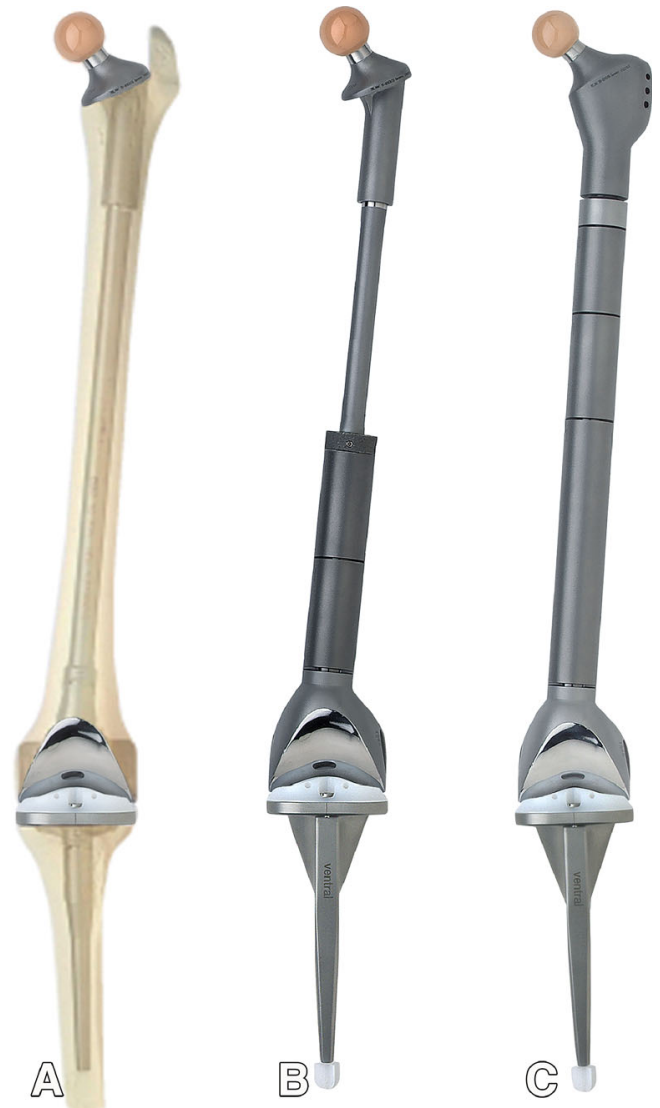

Fig. 1 The concept of the push-through total femoral endoprosthesis and total femoral prosthesis (Megasystem-C, Waldemar Link GmbH \& Co. $\mathrm{KG}$ ). a The push-through total femoral prosthesis (PTTF) allows preservation of muscle attachments on any remaining femoral bone. $\mathbf{b}$ Example of a modular PTTF that preserves the proximal femur. c Example of a total femoral prosthesis (TFP). The proximal part of the prosthesis usually offers a way to attach the hip abductors to the prosthesis

stability and motor control, reduce dislocation rate, allow immediate weight bearing, early rehabilitation and enhance functional outcome.

An analysis of the functional and mechanical performance of the concept of the PTTF has, to our knowledge, never been reported. The few reports that mention PTTFs often group them with TFPs, without assessing this specific concept separately [12]. This study reports the results of the PTTF and assesses this concept as a potential alternative to total femur reconstruction.

\section{Materials and methods}

\section{Study design}

All patients who received a PTTF in two university medical hospitals and one general hospital were included in this retrospective study. All cases that were identified were treated between 2005 and 2014. Patients were excluded if follow-up was sone year.

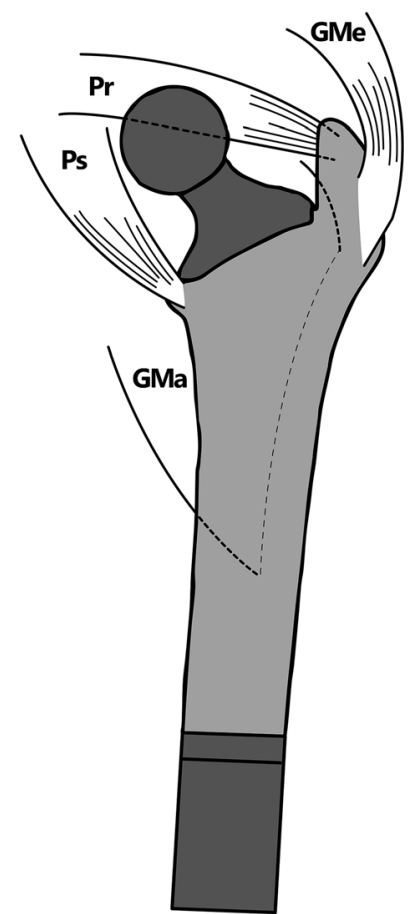

Fig. 2 Advantages of the push-through total femoral prosthesis. This example shows how some of the important hip muscles can be spared in the case that only the most proximal part of the femur is preserved. Only the gluteus medius (GMe), gluteus maximus (GMa), piriformis ( $\mathrm{Pr}$ ), and iliopsoas (Ps) are shown in this example. Others, such as the quadratus femoris, can be spared as well but are not shown in this image. This is not possible when using a total femoral prosthesis

\section{Study procedure}

At baseline, patient characteristics, surgical indication (in case of oncology: oncologic diagnosis and the year of the original diagnosis) and patient history were obtained. All patients were asked to fill out the patient reported outcome measures (PROMs) during a follow-up appointment if no recent questionnaire was present in the existing database.

\section{Surgical procedure}

The surgical approach was subject to variations due to specific patient history and previous surgeries. A patient specific plan was made, based on measurements on a recent CT scan. The patient was positioned in a lateral decubitus position. The hip was approached via the (postero)lateral approach, which was extended along the femur, dorsal of the vastus lateralis muscle. The knee approach was usually midline or anteromedial. In some cases, two separate incisions were used. After preparation of the hip and acetabular components, the knee and tibial components were prepared and placed in situ. A trial reduction was performed with a trial push-through stem into the socket. When this construction proved to be stable and in the right rotation, the definitive prosthesis was assembled 


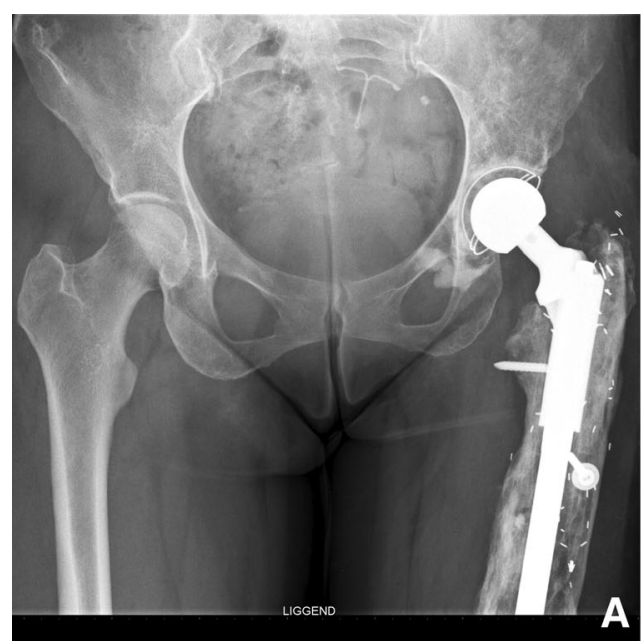

Fig. 3 An example of the push-through prosthesis taken one year postoperatively. a Anterior-posterior radiograph of the pelvis, showing the proximal PTTF and Exeter cup. The push-through stem provides stability after several failed osteosynthesis attempts and a vascularised fibula

and placed. Patients with severe comorbidity were monitored post-operatively in the intensive care unit for 24 hours.

\section{Outcome measures}

The primary outcome measure is the widely used lower limb version of the Musculoskeletal Tumor Society (MSTS) score, which measures functional outcome from the surgeon's point of view; it scores pain, function, emotional acceptance, use of supports, ability to walk and gait on a scale of $0-100 \%$ [13].

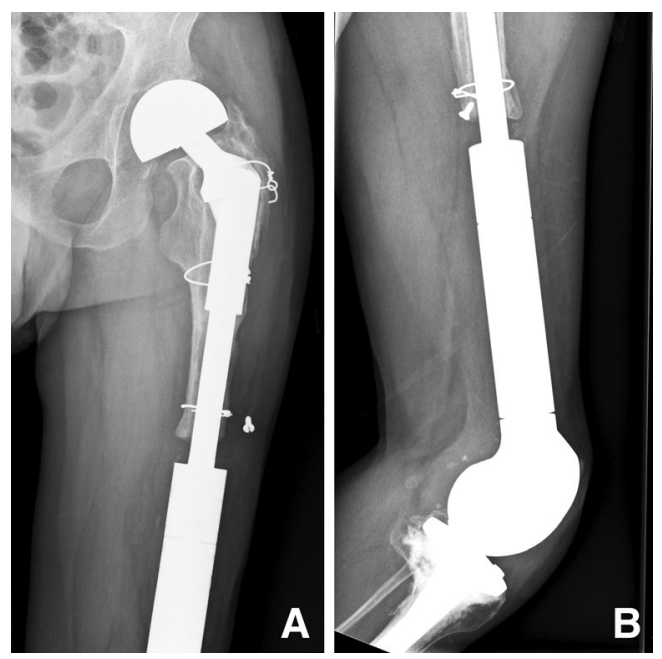

Fig. 4 An example of the push-through prosthesis taken five years postoperatively. a Anterior-posterior radiograph of the left hip and proximal femur. Note how the proximal muscle attachments are spared. Extra cerclage wires are used to improve post-operative fixation of the remaining bone. The Link Variocup is used in this case (hemiarthroplasty). b Lateral radiograph of the rotating-hinge construction of the PTTF. The distal femur has been replaced with a modular push-through construction

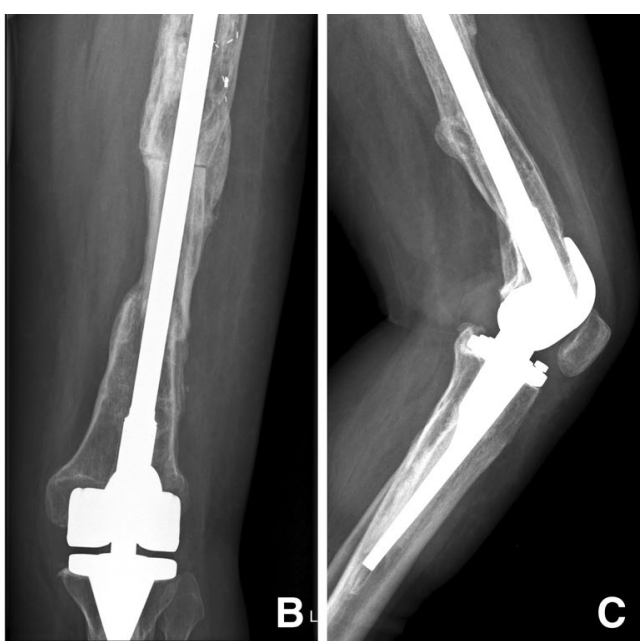

transplant. b Anterior-posterior radiograph of the left distal femur and knee. The distal cortex is spared due to the push-through stem. $\mathbf{c}$ Lateral radiograph of the left knee, showing the rotating hinge knee

Secondary outcome measures (PROMS) feature the numeric rating scale (NRS-11) pain score, NRS fatigue score, NRS satisfaction score, WHO performance status, Toronto Extremity Salvage Score (TESS), EQ-5D-3 L and SF36 health survey. The NRS-11 requires patients to rate their level of pain, fatigue, and satisfaction on a scale from 0 to 10 [14]. The WHO performance status quantifies the patient's ability to function in daily life on a scale from 0 (no impairments) to 5 (death) [15]. The lower limb version of the TESS evaluates physical function and daily activities in patients who suffer from musculoskeletal tumors, on a scale of $0-100 \%$ [16]. The EQ-5D-3L questionnaire (Dutch language, version 1.0) is a standardized health assessment instrument that scores mobility, self-care, usual activities, pain/discomfort and anxiety/ depression [17]. The Dutch version of the SF36 Health Survey is a validated quality of life questionnaire [18].

\section{Other outcome variables}

Peri-operative variables include: duration of surgery, length of stay (LOS), American Society of Anaesthesiologists (ASA) score, and complications (infection, bleeding, transfusion required, thromboembolic events and dislocation).

Patient variables include patient reports, physical examination and range of motion (ROM), survival (and cause of death) and prosthesis failure. Prosthesis failure is defined according to the Henderson classification as: complete revision of endoprosthesis, unplanned revision of a failed part of the prosthesis, fixation of a periprosthetic fracture, soft-tissue reconstruction to improve stability or complete removal of the endoprosthesis without revision or amputation [19]. It is subdivided into type: (1) soft-tissue failure, (2) aseptic loosening, (3) structural failure, (4) infection and (5) tumor 
progression. Complications and peri-operative data up to the removal of the prosthesis were included in the analysis.

\section{Statistical analysis}

Descriptive statistics were used to describe outcome measures. The choice was made to use means and ranges for parametric variables, despite the small sample size, because means are used in virtually all comparative literature. Additionally, medians are reported in the outcome table, to provide a better understanding of the data. Medians with ranges were used for non-parametric data. All data was processed using SPSS version 22.

\section{Ethics}

The medical ethical committee (METc UMCG) declared that ethical testing for this study was not necessary, in accordance with Dutch law. For this type of study formal consent is not required. All actions related to this research were in accordance with the ethical standards of the institutional and/or national research committee and with the 1964 Helsinki declaration and its later amendments or comparable ethical standards.

\section{Results}

Details on inclusion and exclusion are reported in Fig. 5. Baseline characteristics are reported in Table 1. Mean age at

\section{FLOWCHART}

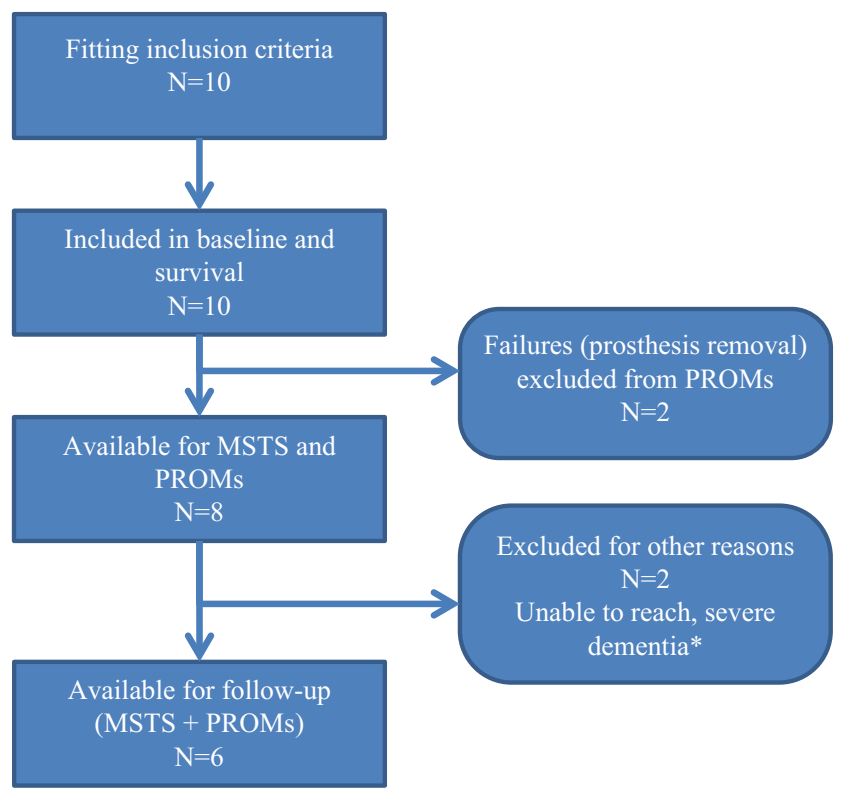

Fig. 5 Study flowchart of inclusion and exclusions. *One patient suffered from severe dementia and therefore was not able to fill out the PROMs surgery was 54 (range 25-77). In seven cases the underlying pathology was oncologic in nature (Table 1).

A modular Megasystem-C (Waldemar Link GmbH \& Co., Hamburg, Germany) endoprosthesis was used in eight cases and a custom made Restoration Modular and MRH/GMRS (Stryker, USA) endoprosthesis was used in two cases. All prostheses featured a rotating-hinge knee. The proximal femur was replaced with the Variocup (Waldemar Link $\mathrm{GmbH} \&$ Co., Hamburg, Germany) (hemiarthroplasty) in five cases. The other five cases received a total hip replacement with a 'dual mobility' Avantage (Biomet, Warsaw, Indiana, USA) cup (3 cases), an Exeter High-wall cup (Stryker, USA) (1 case) and an Exeter Rimfit cup (Stryker, USA) (1 case). Mean follow-up was $5.3(1.1-9.6)$ years.

\section{Primary and secondary outcome}

Primary and secondary outcomes are reported in Table 2. Surgery required 6:33 (4:05-8:46) hours on average. Mean length of stay was 14 (5-41) days. Mean hip flexion was $87^{\circ}\left(45-110^{\circ}\right)$. Mean knee flexion was $88^{\circ}\left(40-110^{\circ}\right)$. During follow-up, there were no radiographic signs of prosthesis loosening in any of the cases.

\section{Oncologic outcome}

All seven oncologic patients were continuously disease-free (CDF) at a mean follow-up of 6.3 (1.1-9.6) years.

\section{Complications}

There were two infections. Both are described in detail under 'Patient and prosthesis survival'. Furthermore, one case of post-operative bleeding required surgical drainage of a large haematoma. Transfusion was required in seven cases due to peroperative blood loss, and in one case to treat pre-existent anaemia. No thromboembolic events or hip dislocation occurred.

\section{Patient and prosthesis survival}

Patient survival was $100 \%$ at a mean follow-up of 5.3 (1.19.6) years. Four prostheses $(40 \%)$ failed in this series: one of which required amputation, one required major revision to a standard TFP, and two required a minor revision. Mean time to failure was 4.4 (1-9) years.

The first failure was due to infection. A female patient with severe rheumatoid arthritis underwent a two-stage revision of her DFP due to loosening, a suspected lowgrade infection with Staphylococcus aureus and a coincidentally-discovered chondrosarcoma in the femur. Cultures of tissue samples obtained during implantation of the custom-made Stryker Restoration Modular PTTF 


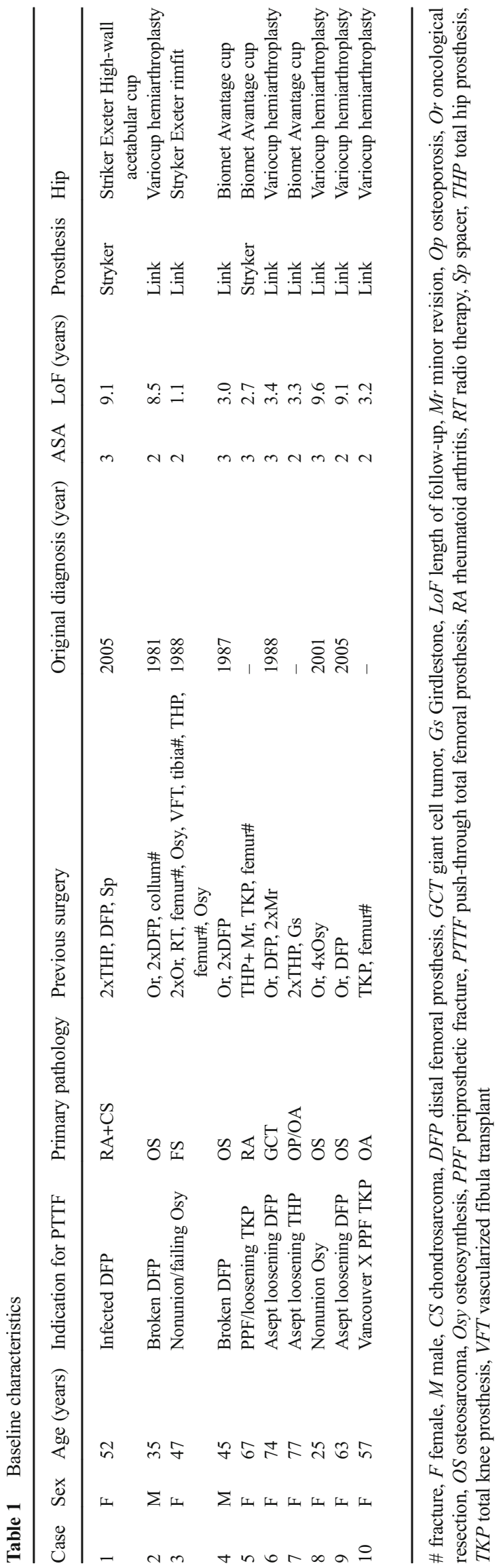

Table 2 Outcome measures

\begin{tabular}{llll}
\hline Outcome measure & Mean & Median & Range \\
\hline MSTS-score (\%) & 64 & 68 & $23-93$ \\
NRS-pain & 1.9 & 0 & $0-5$ \\
NRS-fatigue & 1.8 & 0 & $0-6$ \\
NRS-satisfaction & 8.3 & 8.3 & $6-10$ \\
WHO (frequency of occurring) & & & $1(5)-3(1)$ \\
TESS (\%) & 69 & 79 & $13-90$ \\
EQ-5D & 0.774 & 0.791 & $0.434-1$ \\
SF36 & & & \\
$\quad$ SF36-physical functioning & 48 & 53 & $0-80$ \\
SF36-social functioning & 69 & 75 & $0-100$ \\
SF36-role limit physical & 50 & 50 & $0-100$ \\
SF36-role limit emotional & 72 & 83 & $0-100$ \\
SF36-mental health & 75 & 72 & $64-88$ \\
SF36-vitality & 68 & 70 & $45-90$ \\
SF36-pain & 72 & 79 & $10-100$ \\
SF36-general health & 67 & 65 & $60-80$ \\
SF36-health change & 29 & 25 & $0-50$ \\
\hline
\end{tabular}

MSTS Musculoskeletal Tumor Society score, NRS numeric rating scale, WHO World Health Organisation performance scale, TESS Toronto Extremity Salvage Score, EQ-5D-3L EuroQol health-related quality of life survey, SF36 Short Form 36 health survey. Both the mean and median are listed for variables that usually fit a normal distribution because due to the small sample size, the mean is sensitive for outliers.

were negative. The low-grade periprosthetic infection with Pseudomonas aeruginosa, which arose after three years, resulted in surgical debridement and hip disarticulation after five years of suppressive antibiotics.

The second failure was due to the material. The connection between the MRH knee prosthesis and the custom-made Stryker Restoration Modular femoral stem broke after almost three years, during walking. It was revised to a Stryker GMRS total femoral prosthesis.

The third failure was due to a spontaneous infection, five years after receiving the Link Megasystem-C PTTF. The periprosthetic infection was treated with i.v. antibiotics, surgical debridement of the wound and local gentamicin beads. Eventually, all polyethylene parts of the PTTF were replaced and all metal parts were cleaned and re-implanted. Persistent wound problems required a gastrocnemius flap and skin transplant. The patient is now infection free and doing well, three years after finishing the antibiotic treatment.

The fourth failure was due to persistent pain after receiving the Link Megasystem-C PTTF. It was attributed to unsuccessful adherence of the proximal femoral bone to the PTTF, an internal rotation contracture and insufficient anteversion. It was regarded as a structural failure. The anteversion angle was corrected and the proximal femoral bone was cemented onto the prosthesis during a minor revision one year after initial implantation. 


\section{Discussion}

A push-through total femoral endoprosthesis saves part of the bone and its muscle attachments and most likely preserves its stability and function better than a TFP. A retrospective analysis was performed to assess the outcome of the PTTF. All patients had a long medical history, severe comorbidity and extensive previous surgeries. All patients survived, but the complication rate was substantial. Functional outcome varied from severely impaired to virtually no impairment, however, almost all patients had relevant disabilities. Emotional functioning, perceived health and overall quality of life were less impacted and most patients had little to no pain. Satisfaction with the result of this procedure was very high. There were no hip dislocations.

Mean MSTS score in this study was $64 \%$, which is comparable to mean MSTS scores after primary TFPs (50-73\%) $[4,8,20]$. Secondary TFPs might result in lower MSTS scores than primary TFPs (60\% versus $73 \%$ ) [10]. MSTS scores were positively influenced by the lack of use of pain medication; nevertheless, functional impairment and gait decreased the scores. Individual scores showed great variability (23-93\%), similar to TFP outcome $[8,20]$. A similar spread is seen in the TESS scores (13-90\%). Mean TESS scores after TFP range from $47 \%$ to $69 \%$ in the literature, with equally large interpatient variability [7, 21]. A mean TESS score of $69 \%$ after PTTF is relatively high.

All patients have functional impairments to some degree after total femoral prostheses; five patients had a WHO performance scale of 1 (only limited in heavy exercise) and had an EQ5D index higher than 0.7. One patient had a WHO performance scale of 3 (limited self-care and $>50 \%$ of the day in bed) and a lower EQ5D score (0.434). 'Usual activities' and 'mobility' impacted the EQ5D index to the greatest extent, while 'selfcare', 'anxiety/depression' and 'pain/discomfort' played a smaller role. The low levels of pain are also seen in the NRS pain scores and the SF36 pain sub-scores. Furthermore, four patients experienced no lasting fatigue after the surgery. Two patients experienced fatigue but were very satisfied with their outcome. Many patients mentioned their gratitude for the attempts to spare the leg - as well as the success achieved - and took the alternative option (amputation) into account in their satisfaction scores. Satisfaction scores are surprisingly high despite the functional impairment. The SF36 shows impaired physical functioning (mean 48) and clear limitations (mean 50 ) due to impaired function. General health perception (mean 67 ) and perceived vitality (mean 68) show similar scores.

Range of motion plays an important role in the physical functioning of the limb. Mean hip flexion of $87^{\circ}$ and knee flexion of $88^{\circ}$ after PTTF is higher than after TFP: knee flexion between $60^{\circ}$ and $73^{\circ}$ (range $0-120^{\circ}$ ) and hip flexion of $61^{\circ}$ has been reported $[4,22]$. The theoretical advantages of preserved muscle attachments of the PTTF could explain this; nevertheless, more and other types of studies are necessary to evaluate this.

\section{Complications}

In general, complications were comparable to the series by Clement et al. [5], who reported 19 cases of TFP after periprosthetic fractures [5].

Our infection rate $(20 \%)$ is similar to the reported 10 $22 \%$ infections after TFP $[4-6,8]$. One patient had a previous infection of her DFP, however, this is not a definite contraindication [10].

Hip dislocations occur often after TFP (5.3-23\%), though none occurred in our study $[5-8,10]$. Our small sample size could be a factor; however, other factors might explain this. Excision of the hip abductor muscles increases dislocation rates of TFPs, something that is averted in the PTTF [7, 10]. Furthermore, all PTTFs were used as secondary limbsalvaging treatment, which is linked to lower dislocation rates in TFPs compared to primary limb salvaging TFPs ( $9 \%$ versus 23\%) [10]. Additionally, a dual-mobility Variocup and the Avantage cup were used in most hemiarthroplasties and THPs respectively, which is known to reduce dislocation rates in both primary and revision THAs and hemiarthroplasties [23]. Other studies are necessary to assess these factors.

Aseptic loosening often complicates DFPs, however, implementation of rotating hinge prostheses instead of fixedhinge prostheses has reduced this significantly [24]. In this study only rotating hinge prostheses were used.

\section{Failures}

Failure rate in this study is high (40\%), although this might be partly influenced by our broad definition of failure. Sewell et al. reported five-year TFP survival of $100 \%$ considering removal as the endpoint, which dropped to $56 \%$ with re-operation as endpoint [10]. Our study showed $80 \%$ prosthesis survival and $90 \%$ limb-survival, which is very satisfying given the type of conditions treated. There were too few patients in our study to draw valid conclusions about the differences between the Link and Stryker techniques. However, a modular design has the advantage that it allows the surgeon to adjust to factors that could not have been foreseen during pre-operative planning.

\section{Patient analysis}

It is relevant to note that the lowest functioning patient was impaired by comorbidity (myocardial infarction, rheumatoid arthritis, depression) to a far greater extent than by the outcome of the PTTF, which means her scores might not accurately reflect PTTF outcome. The patient with the worst ROM (hip flexion $45^{\circ}$, knee flexion $40^{\circ}$ ) previously underwent resection of the knee extensors and partial reconstruction by transposition of the biceps femoris muscle during primary oncologic resection and 
patellar resection during revision DFP surgery. All other patients had a knee and hip flexion of more than $90^{\circ}$ in our study, which is remarkably good.

The two highest functioning patients both were relatively young, had relatively mild comorbidity and fewer previous surgical procedures (i.e. less soft tissue damage) than most other patients. One patient has a job in the food service industry that requires mostly standing and walking for eight hours a day without problems.

The result of any megaprosthesis will probably improve when patients have had fewer previous surgery; nevertheless, the push-through prosthesis might have more to gain than the TFP because it preserves muscle function while providing the same structural integrity to the femur (Fig. 2). The analysis seems to suggest that the PTTF might be suited for patients with limited soft-tissue damage and sufficient femoral bone stock, while patients with severe soft-tissue damage (i.e. resection of the extensor apparatus) and comorbidity reap none of the benefits and might be better off with a TFP. However, larger studies with longer follow-up will provide more evidence.

Oncologic outcome in the present patients was good and patient survival was $100 \%$. Studies on TFPs often report patient survival rates as low as $32-35 \%$, two to five years after primary tumor resection and limb-salvaging with a TFP [10, 20]. Recurrent disease occurs less often after secondary limb-salvaging surgery [10]. All seven oncologic patients received a PTTF as a secondary treatment because they survived long enough to develop complications of their primary surgery. This inherently selects patients with favourable oncologic outcome.

This patient selection bias (both PTTFs and TFPs were used in the study period) is one of the limitations of this study. Furthermore, the sample size is small because this prosthesis is rarely used. However, all patients who received a PTTF between 2005 and 2014 were included, thus reducing the sampling bias for PTTF patients. Moreover, there is no control group with which to compare the findings. Due to the nature of the prosthesis as a 'last option' for limb-salvaging there is great heterogeneity among the patients. The length of the follow-up varies, making it difficult to note time-dependent changes in the outcome measures. In spite of these restrictions, this is the first study to report specifically on the push-through prosthesis.

In summary, patient satisfaction was very high, despite that functional outcome ranges from severely handicapped to near-normal functioning. Complication rate was within the ranges that are reported in the literature. However, no dislocations occurred. Only one complication resulted in hip disarticulation. The modular PTTF offers the surgeon a good alternative to TFP due to its highly patient-specific approach, preservation of muscle attachments and good functional outcome.
Acknowledgements We thank Link GmbH \& Co. KG for providing us with the images of the Megasystem-C prostheses. Furthermore, we thank A.S. de Wit for designing the conceptual image of the push-through prosthesis and the preserved muscle attachments.

\section{Compliance with ethical standards}

Conflict of interest The corresponding author (Gorter) has received partial support for travel and accommodation for the NOF Congress from Link GmbH \& Co. KG, in accordance with the 'Code of Conduct Medical Devices'.

All other authors declare that they have no competing interests.

Funding This research did not receive any grants from funding agencies in the public, commercial, or not-for-profit sectors.

Open Access This article is distributed under the terms of the Creative Commons Attribution 4.0 International License (http:// creativecommons.org/licenses/by/4.0/), which permits unrestricted use, distribution, and reproduction in any medium, provided you give appropriate credit to the original author(s) and the source, provide a link to the Creative Commons license, and indicate if changes were made.

\section{References}

1. Bacci G, Longhi A, Fagioli F, Briccoli A, Versari M, Picci P (2005) Adjuvant and neoadjuvant chemotherapy for osteosarcoma of the extremities: 27 year experience at Rizzoli Institute, Italy. Eur J Cancer 41:2836-2845. doi:10.1016/j.ejca.2005.08.026

2. Grimer RJ (2005) Surgical options for children with osteosarcoma. Lancet Oncol 6:85-92. doi:10.1016/s1470-2045(05)01734-1

3. Ando K, Heymann MF, Stresing V, Mori K, Redini F, Heymann D (2013) Current therapeutic strategies and novel approaches in osteosarcoma. Cancers (Basel) 5:591-616. doi:10.3390/ cancers5020591

4. Ahmed AR (2010) Total femur replacement. Arch Orthop Trauma Surg 130:171-176. doi:10.1007/s00402-009-0945-2

5. Clement ND, MacDonald D, Ahmed I, Patton JT, Howie CR (2014) Total femoral replacement for salvage of periprosthetic fractures. Orthopedics 37:e789-e795. doi:10.3928/01477447-20140825-55

6. Friesecke C, Plutat J, Block A (2005) Revision arthroplasty with use of a total femur prosthesis. J Bone Joint Surg Am 87:26932701. doi:10.2106/jbjs.d.02770

7. Jones KB, Griffin AM, Chandrasekar CR, Biau D, Babinet A, Deheshi B, Bell RS, Grimer RJ, Wunder JS, Ferguson PC (2011) Patient-oriented functional results of total femoral endoprosthetic reconstruction following oncologic resection. J Surg Oncol 104: 561-565. doi:10.1002/jso.22003

8. Natarajan MV, Balasubramanian N, Jayasankar V, Sameer M (2009) Endoprosthetic reconstruction using total femoral custom mega prosthesis in malignant bone tumours. Int Orthop 33:13591363. doi:10.1007/s00264-009-0737-x

9. Morris HG, Capanna R, Campanacci D, Del Ben M, Gasbarrini A (1994) Modular endoprosthetic replacement after total resection of the femur for malignant tumour. Int Orthop 18:90-95. doi:10.1007/ bf02484417

10. Sewell MD, Spiegelberg BG, Hanna SA, Aston WJ, Bartlett W, Blunn GW, David LA, Cannon SR, Briggs TW (2009) Total femoral endoprosthetic replacement following excision of bone tumours. J Bone Joint Surg (Br) 91:1513-1520. doi:10.1302/0301620x.91b11.21996 
11. Gosheger G, Hillmann A, Lindner N, Rodl R, Hoffmann C, Burger H, Winkelmann W (2001) Soft tissue reconstruction of megaprostheses using a trevira tube. Clin Orthop Relat Res 393: 264-271

12. Ham SJ, Schraffordt Koops H, Veth RP, van Horn JR, Molenaar WM, Hoekstra HJ (1998) Limb salvage surgery for primary bone sarcoma of the lower extremities: long-term consequences of endoprosthetic reconstructions. Ann Surg Oncol 5:423-436. doi: 10.1007/bf02303861

13. Enneking WF, Dunham W, Gebhardt MC, Malawar M, Pritchard DJ (1993) A system for the functional evaluation of reconstructive procedures after surgical treatment of tumors of the musculoskeletal system. Clin Orthop Relat Res 286:241-246

14. Ferreira-Valente MA, Pais-Ribeiro JL, Jensen MP (2011) Validity of four pain intensity rating scales. Pain 152:2399-2404. doi:10. 1016/j.pain.2011.07.005

15. World Health Organization (1979) WHO handbook for reporting results of cancer treatment. World Health Organization, Geneva

16. Davis AM, Wright JG, Williams JI, Bombardier C, Griffin A, Bell RS (1996) Development of a measure of physical function for patients with bone and soft tissue sarcoma. Qual Life Res 5:508-516. doi:10.1007/bf00540024

17. EuroQol Group (1990) EuroQol - a new facility for the measurement of health-related quality of life. Health Policy 16:199-208. doi:10.1016/0168-8510(90)90421-9

18. Aaronson NK, Muller M, Cohen PD, Essink-Bot ML, Fekkes M, Sanderman R, Sprangers MA, te Velde A, Verrips E (1998)
Translation, validation, and norming of the dutch language version of the SF-36 health survey in community and chronic disease populations. J Clin Epidemiol 51:1055-1068. doi:10.1016/s08954356(98)00097-3

19. Henderson ER, Groundland JS, Pala E, Dennis JA, Wooten R et al (2011) Failure mode classification for tumor endoprostheses: Retrospective review of five institutions and a literature review. J Bone Joint Surg Am 93:418-429. doi:10.2106/jbjs.j.00834

20. Ruggieri P, Bosco G, Pala E, Errani C, Mercuri M (2010) Local recurrence, survival and function after total femur resection and megaprosthetic reconstruction for bone sarcomas. Clin Orthop Relat Res 468:2860-2866. doi:10.1007/s11999-010-1476-4

21. Tunn PU, Pomraenke D, Goerling U, Hohenberger P (2008) Functional outcome after endoprosthetic limb-salvage therapy of primary bone tumours-a comparative analysis using the MSTS score, the TESS and the RNL index. Int Orthop 32:619-625. doi: 10.1007/s00264-007-0388-8

22. Ward WG, Dorey F, Eckardt JJ (1995) Total femoral endoprosthetic reconstruction. Clin Orthop Relat Res 316:195-206

23. De Martino I, Triantafyllopoulos GK, Sculco PK, Sculco TP (2014) Dual mobility cups in total hip arthroplasty. World J Orthop 5:180187. doi:10.5312/wjo.v5.i3.180

24. Myers GJ, Abudu AT, Carter SR, Tillman RM, Grimer RJ (2007) Endoprosthetic replacement of the distal femur for bone tumours: long-term results. J Bone Joint Surg (Br) 89:521-526. doi:10.1302/ 0301-620x.89b4.18631 